\title{
Highly optimized tolerance in epidemic models incorporating local optimization and regrowth
}

\author{
Carl Robert* and J. M. Carlson \\ Department of Physics, University of California, Santa Barbara, California 93106 \\ John Doyle \\ Control and Dynamical Systems, California Institute of Technology, Pasadena, California 91125
}

(Received 29 August 2000; published 25 April 2001)

\begin{abstract}
In the context of a coupled map model of population dynamics, which includes the rapid spread of fatal epidemics, we investigate the consequences of two new features in highly optimized tolerance (HOT), a mechanism which describes how complexity arises in systems which are optimized for robust performance in the presence of a harsh external environment. Specifically, we (1) contrast global and local optimization criteria and (2) investigate the effects of time dependent regrowth. We find that both local and global optimization lead to HOT states, which may differ in their specific layouts, but share many qualitative features. Time dependent regrowth leads to HOT states which deviate from the optimal configurations in the corresponding static models in order to protect the system from slow (or impossible) regrowth which follows the largest losses and extinctions. While the associated map can exhibit complex, chaotic solutions, HOT states are confined to relatively simple dynamical regimes.
\end{abstract}

DOI: 10.1103/PhysRevE.63.056122

PACS number(s): 05.65.+b, 05.45. $-\mathrm{a}, 87.23 .-\mathrm{n}$

\section{INTRODUCTION}

The property of agent based modeling of complex systems which has been most widely emphasized has been the emergence of complex behavior from spatiotemporal dynamical systems described by simple local rules. In many of the most widely studied examples, complexity is associated with system wide, self-organization to a critical point [selforganized criticality (SOC) [1,2]] or a bifurcation point near the "edge of chaos" [3]. In both cases, even the generic, random states exhibit long range correlations. However, with the exception of power law statistics, properties associated with random configurations fail to capture the basic attributes of most natural and man-made complex systems, where evolution and deliberate design lead to more regular structure and higher densities than are achieved randomly [4]. On the other hand, it has been difficult to develop models which capture the emergence of higher level structure, so that for most examples which have been studied in this context, design features are put in by hand [5-8].

Recently, highly optimized tolerance (HOT) [9-11] was introduced as a mechanism for complexity, in which nongeneric features do emerge without being introduced directly. Instead, they result from optimization of a design objective in systems consisting of many internal degrees of freedom coupled to an uncertain external environment. HOT emphasizes robustness to external perturbations as the key mechanism which can lead to structured, high-density configurations. HOT leads to power laws, and, more importantly, to systems which are robust to common, designed-for uncertainties, and fragile to design flaws and rare perturbations.

While the basic characteristics associated with HOT are shared by a wide variety of systems in engineering, biology,

\footnotetext{
*Author to whom correspondence should be addressed. Email address: crobert@physics.ucsb.edu
}

and ecology, the deliberate static design methodologies based on systemwide performance objectives which have been used in all of the studies to date are much more clearly connected to man-made technologies than to systems which arise in nature. In ecological and biological settings the distinction between a system and its environment is typically more ambiguous and involves higher levels of feedback, and the question of the scale on which natural selection, and thus evolution, acts is a matter of considerable debate [4,12-15].

In this paper we address two issues of key importance to biological and ecological applications which were not treated in the initial investigations of HOT. We still focus on the simplest possible settings, here consisting of coupled maps motivated by spatiotemporal models of population dynamics subject to external disturbances (infections). While the maps can exhibit high period orbits and chaotic solutions, HOT states are associated with much simpler solutions.

In Sec. II we provide a brief review of HOT, focusing on issues which are relevant for the work presented here and developing a context for our application in epidemics modeling. In Sec. III we compare configurations obtained from global and local optimization of static fitness. In the context of ecology, global optimization loosely corresponds to optimization of fitness on the scale of an ecosystem as a whole, while local optimization corresponds to some lower level optimization on the scale of individual organisms or groups of organisms. In general, local and global optimization need not yield the same configurations (though in our highly simplified setting quite often they do). However, both local and global optimization do generally lead to rare, structured states which differ significantly from generic random configurations, and are both robust to common events, and highly sensitive to changes in the pattern of disturbance.

In Sec. IV we move beyond studies of static configurations subject to a single epidemic and incorporate regrowth of the population through an explicitly time dependent map. We summarize the behaviors of the model in terms of a 
phase diagram. In the deterministic case parameter space is divided into distinct dynamical regimes which include stable fixed points, stable periodic orbits, and chaos. We also consider stochastic driving, which leads to moderately higher performance compared to the corresponding deterministic case as well as simpler behavior dynamically. While optimal solutions are always associated with simple stable fixed point solutions, the threat of slow regrowth or extinction following a rare event leads the system away from the static optima, where higher initial densities lead to larger losses. Such large losses would result in extended yield penalties during the growth phase relative to the state which is optimized including the dynamics. Finally, we conclude in Sec. V with a discussion of potential avenues for future applications of our results.

\section{HIGHLY OPTIMIZED TOLERANCE}

In this section we provide a brief review of earlier results describing HOT in the context of percolation models, which serves as a starting point for the work described here $[9,10]$. Highly optimized tolerance was initially introduced in the context of the familiar percolation [16], forest fire [17], and sandpile [1] models studied in the context of self-organized criticality [2]. Each of these models consists of a lattice of sites which can be occupied by one (percolation and forest fire models) or more (sand pile models) particles. The lattice is also subject to local disturbances, which may ultimately remove particles from the system via a cascading failure event, described by local rules for the propagation of failure in the system. The new ingredient associated with HOT is the introduction of deliberate design or evolution by natural selection [11] to these models. Design and evolution favor high yield configurations. Here yield corresponds to the average density of particles which remains in the system after a failure event. In a variety of different scenarios it has been shown that HOT states have densities well in excess of the corresponding models at criticality. HOT states exhibit power laws, but with exponents which are steeper than those at criticality. Unlike criticality, HOT is associated with modular patterned states, corresponding to a set of measure zero in the space of possible configurations at any density. HOT states are robust to common perturbations, but are especially sensitive, or fragile, to design flaws and rare or unanticipated perturbations.

The example which most directly relates to the work described in this paper is the standard percolation model, modified to include propagating failure events. This model has been studied previously by others in a variety of contexts, including fuel limited forest fires and contagious epidemics propagating among stationary organisms. In this paper we focus on issues which arise in developing applications of HOT to the study of population dynamics in ecological systems. Thus we will use the language of epidemics. However, our model is sufficiently general that various alternative analogies would be equally relevant.

In the standard percolation model sites on a lattice are independently occupied by organisms with probability $\rho$ and vacant with probability $1-\rho$. The population density on the lattice is thus equal to $\rho$, and any configuration with density $\rho$ is equally likely. If an infection strikes a site on the lattice, disease spreads through the connected cluster of nearest neighbor occupied sites, killing all of the organisms in the cluster. Thus, for a given initial starting density the average density of organisms which survives the epidemic is the yield, $Y=\rho-\langle$ loss $\rangle$, where the angle brackets represent an average over both the distribution of initial infection sites and the ensemble of possible configurations at density $\rho$. For the standard percolation model, the configuration at any given density is random, so that in the limit of an infinite system, the probability distribution describing the relative likelihood of infection striking different sites on the lattice is irrelevant.

In the standard percolation model in the thermodynamic limit, a plot of $Y$ vs $\rho$ increases linearly and monotonically over the range $Y=\rho=0$ up to the critical point $Y=\rho=\rho_{c}$, followed by a monotonic decrease over the range $\rho_{c} \leqslant \rho \leqslant 1$. At densities below criticality individual infections never lead to a macroscopic loss. Instead the typical loss cuts off at the size corresponding to the correlation length, which does not scale with the size of the system. For densities above the critical point, the characteristic loss is of order the system size, and is associated with infections which hit and spread through the infinite cluster. In general, yield is related to the percolation probability $P_{\infty}(\rho)$ :

$$
Y=\left[1-P_{\infty}(\rho)\right] \rho+P_{\infty}(\rho)\left[\rho-P_{\infty}(\rho)\right] .
$$

Here the first term corresponds to the probability that the initial infection misses the infinite cluster, in which case the full initial density is retained. The second term corresponds to the probability that the infections strikes a site in the infinite cluster, in which case the loss in density incurred is that associated with the infinite cluster.

For $\rho_{c} \leqslant \rho \leqslant 1, P_{\infty}(\rho)$ increases monotonically from zero to one, with $P_{\infty}(\rho) \sim\left(\rho-\rho_{c}\right)^{\beta}$ in the limit $\rho \rightarrow \rho_{c}+[16]$. For $\rho=1$ the yield is (trivially) $Y=0$ because the infection spreads throughout the system. In all dimensions, the maximum yield occurs for $\rho=\rho_{c}$, which is the maximum density at which the system sustains no macroscopic loss. This behavior is qualitatively well captured by the mean-field-like form, which we assume describes the percolation probability as a function of density, throughout the full range of densities, for an ensemble of random configurations:

$$
P_{\infty}(\rho)= \begin{cases}0, & 0 \leqslant \rho \leqslant \rho_{c} ; \\ {\left[\left(\rho-\rho_{c}\right) / \rho_{c}\right]^{1 / 2},} & \rho_{c} \leqslant \rho \leqslant 1 .\end{cases}
$$

We set $\rho_{c}=1 / 2$, based (loosely) on bond percolation in two dimensions. The specific choice of $\rho_{c}$ does not significantly alter our results. The corresponding yield is simply given by a tent function

$$
Y= \begin{cases}\rho, & 0 \leqslant \rho \leqslant \rho_{c} \\ 1-\rho, & \rho_{c} \leqslant \rho \leqslant 1 .\end{cases}
$$

HOT configurations optimize the yield for systems subject to a particular distribution of infections $P(i, j)$ and 
specified constraints on the optimization procedure. In the case of percolation, if the only design parameter is the density $\rho$ of the initial configuration, the maximum yield occurs at density $\rho_{c}$. However, if more degrees of freedom are allowed in determining the optimal design, specialized configurations can be chosen that produce maximal yields $Y$ $=\rho$ all the way up to the maximum density $\rho=1$. This was studied previously in the context of a forest fire analogy on a two-dimensional square lattice [9], where large numbers of design degrees of freedom led to highly stylized, modular configurations, which, unlike the random case, were specially sensitive to the distribution of sparks $P(i, j)$. In particular, HOT configurations are robust to common perturbations, and fragile with respect to rare perturbations and changes in the distributions of hits.

In the context of population dynamics, a HOT configuration corresponds to optimal spatial clustering of organisms, which maximizes the survival of the population in the presence of external infections. Deliberate design could enter into the problem in the case of a managed community of organisms (e.g., a farm), where optimal yield would relate directly to profit. Alternatively, in a natural community high yield configurations correspond to communities which attain high densities of biomass through specialized traits which arise through evolutionary processes [4,18].

However, considering evolutionary processes acting on a community of organisms introduces many issues which were not taken into account in the initial studies of static systems subject to deliberate design. In the following sections of this paper we will begin to address some of the key issues that fall into this category. The first is the scale on which evolution and selection acts. Namely, how do results obtained for systems which are optimized as a whole compare to systems in which different regions (or species or organisms) are optimized individually for their own best outcome? Second, what is the role of time dependent regrowth on the optimal configuration? That is, if the full growth cycle is included in the estimate of yield, then there may be an additional penalty associated with rare perturbations due to the long period required for regeneration. These are the two issues which we study in the remaining sections of this paper in the context of a simple dynamical map.

\section{GLOBAL AND LOCAL OPTIMIZATION IN A COUPLED MAP REPRESENTATION OF A POPULATION SUBJECT TO EPIDEMICS}

We construct a low-dimensional map which corresponds to the coarse graining of an infinite underlying system. This map is based on mean-field-like percolation results described in the previous section. It is defined by a set of functions describing the expected size of an epidemic which spreads within a population of organisms following the infection of an individual site. Compared to previous studies of HOT, here we consider a very limited number of design degrees of freedom, which will represent the degrees of freedom of our dynamical system. In particular, we consider systems of $N$ $=1,2$, and 3 designable spatial degrees of freedom. Each of these degrees of freedom can be thought of as a cell (see Fig. (a)

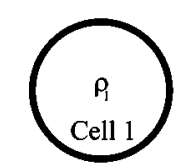

(b)

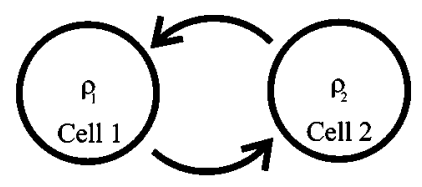

(c)

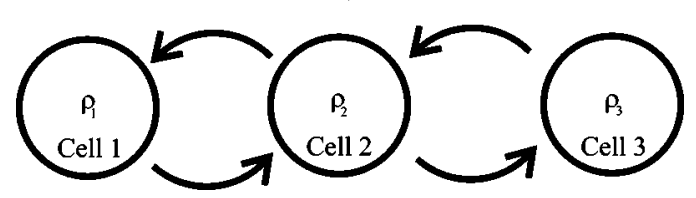

FIG. 1. Schematic representation of the coupled map model for (a) $N=1$, (b) $N=2$, and (c) $N=3$ cell cases. Each cell is characterized by a density $\rho_{i}$, and has a probability of infection $p_{i}$. Epidemics can spread between nearest neighbor cells, as represented by the arrows.

1 ) which is characterized by a density $\rho_{i}, i=1, \ldots, N$. Here the word "cell" describes a region containing many (essentially infinite) organisms, and the loss within each cell is described by the traditional percolation model of the previous section, along with a coupling between cells which we describe below. That is, we assume each "cell" is characterized by its density $\rho_{i}$, so that sites within each cell are independently occupied with probability $\rho_{i}$, and that properties of the cell are given by the ensemble average at that density. We also assume there is a probability $p_{i}$ of an infection striking within cell $i$, such that the probability of hitting one of the $N$ cells sums to unity. All structure in the distribution of infections which might exist at a resolution which exceeds that which defines the boundary of the particular cell which is infected is irrelevant (as in random percolation), because of the ensemble average of random configurations within each cell which is used to determine losses within the cells.

In our study, parameters of the dynamical system may be tuned to maximize the population density after an infection. The model is sufficiently general that it could be motivated by other applications (e.g., toy forests subject to fires). When an infection strikes within a cell, some of the density may be lost, and the loss may also spread into neighboring cells, leading to additional damage. We focus on the yield $Y$, a measure of the fitness, defined to be the density remaining after an epidemic. For our comparisons between global and local optimization of the fitness we distinguish between the systemwide global yield $Y=N^{-1} \sum_{i=1}^{N} Y_{i}$, and the local yield $Y_{i}$ within each cell. Several previous studies of HOT have focused on generalizations of percolation and forest fire models which incorporate design of the configuration in a manner which optimizes yield for a given distribution of sparks. The essential feature of these models, preserved here, is the tradeoff between high densities, necessary for high 
yields, versus low densities for protection against the spread of infections.

The only macroscopic loss in density is associated with events involving an infinite connected cluster. At density $\rho_{i}$, if an infection hits within cell $i$ (which occurs with probability $p_{i}$ ), the probability of hitting an infinite cluster is given by the percolation probability, Eq. (2), with $\rho=\rho_{i}$. As before, we set $\rho_{i}=\rho_{c}=1 / 2$ to be the critical density, associated with the emergence of an infinite cluster in cell $i$. Qualitatively our results are insensitive to the particular value chosen for $\rho_{c}$.

The corresponding expected yield $Y_{i}$ within cell $i$ is given by Eq. (1), which simplifies slightly:

$$
Y_{i} \mid(\text { hit in } i)=\rho_{i}-P_{\infty}^{2}\left(\rho_{i}\right) .
$$

For the one cell case, $p_{1}=1$, and this completely defines the static yield map as a function of the initial density $\rho_{1}$. For two or three cells, this also describes the loss in an individual cell when it is hit. However, in the two and three cell cases we must also include terms describing the propagation of epidemics between cells, which we assume is described by the same underlying mean-field-like percolation mechanism. When an infection strikes cell $i$, the probability the epidemic propagates into a nearest neighbor $j=i \pm 1$, is given by the percolation probability in the hit cell $P_{\infty}\left(\rho_{i}\right)$. When spreading occurs, the loss in cell $j$ is then calculated in a manner which parallels Eq. (4), as if $j$ were hit. Here the mean field assumption implies that loss in cell $j$ depends only on the fact that the epidemic has spread from cell $i$ into $j$ (so that $j$ is also impacted by the epidemic), and not on terms (associated with finite-dimensional unstirred systems) which would distinguish between the spread of disease from an arbitrary site in the cell vs. spread which is initiated at the boundary of the cell. This leads to

$$
Y_{j=i \pm 1} \mid(\text { hit in } i)=\rho_{j}-P_{\infty}\left(\rho_{i}\right) P_{\infty}^{2}\left(\rho_{j}\right) .
$$

This combined with Eq. (4) completes the $N=2$ map.

Finally, in the case $N=3$ we also need to consider second nearest neighbors (cells 1 and 3 are second neighbors of each other, but cell 2 does not have a second neighbor). The probability that an epidemic will propagate into cell $k$, which is displaced two cells from the hit cell $(k=i \pm 2)$, is given by the product of the percolation probability in the initial cell, and the cell $j$ which is intermediate between $i$ and $k$. This leads to

$$
Y_{k=i \pm 2} \mid(\text { hit in } i)=\rho_{k}-P_{\infty}\left(\rho_{i}\right) P_{\infty}\left(\rho_{j}\right) P_{\infty}^{2}\left(\rho_{k}\right) .
$$

These yield maps are written explicitly for the separate cases $N=1,2,3$ in the Appendix.

Next we compare global and local results for the static optimization of the yield. We begin with the case $N=1$, which is trivial, but included here for completeness. In this case, there is only one degree of freedom for the design - the initial density $\rho_{1}$ of the cell. Thus there is a priori no distinction between global and local optimization. A plot of $Y=Y_{1}$ as a function of $\rho=\rho_{1}$ for this case has a single maximum at $\rho=1 / 2$, the percolation threshold. This is the maxi- mum density at which the system can be hit, yet sustain no macroscopic loss. At lower initial densities, there is also no loss, but the yield is lower because of the lower initial $\rho_{1}$. At higher initial densities, the yield is less because of the cost associated with the finite probability of hitting the infinite cluster. This is simply a reiteration of the results we discussed for random percolation in Sec. II.

For the case $N=2$, we define $p_{1}=1-p$, and $p_{2}=p$. Without loss of generality we take $p \leqslant 1 / 2$, so that cell one is the cell which is more likely to be hit. For the global case we adjust the densities $\rho_{1}$ and $\rho_{2}$ to produce the maximum global $Y=\left(Y_{1}+Y_{2}\right) / 2$. We find that the maximum $Y$ always corresponds to $\rho_{1}=1 / 2$, and $\rho_{2}=1$ (see Appendix for details). The optimal fitness of the system is attained when the cell which is most likely to be hit is at the percolation threshold. As previously noted for the case $N=1$, this corresponds to the maximum density for which there is no macroscopic loss in the hit cell. In the case of two or more cells, propagation between cells is also relevant. At density $\rho=1 / 2$, the absence of an infinite cluster guarantees that the probability of an epidemic spreading (macroscopically) is zero. On the other hand, it is more surprising that the cell which is less likely to be hit is fully occupied, even for $p$ very close to $1 / 2$ (in which case there is only a small difference in the probabilities for the two cells). Unit occupation density guarantees that the less likely cell suffers a complete loss (extinction) when it is hit.

A plot of $Y$ as a function of $\rho_{1}$ and $\rho_{2}$ reveals a single maximum with a value of $Y=(3 / 2-p) / 2$ at $\left[\rho_{1}, \rho_{2}\right]$ $=[1 / 2,1]$. The maximum becomes increasingly steep as $p$ decreases. When $p=1 / 2$ the configurations $\left[\rho_{1}, \rho_{2}\right]$ $=[1 / 2,1 / 2],[1 / 2,1],[1,1 / 2]$ become degenerate. These results for global optimization of the two cell case are derived analytically in the Appendix, and are in agreement with recent simulations of discrete lattice models with two tunable density parameters, to be presented elsewhere [19].

Local optimization requires that the densities within each cell be adjusted in a manner which maximizes only the local fitness within the given cell. Interestingly, in the case of two cells for all $p,\left[\rho_{1}, \rho_{2}\right]=[1 / 2,1]$ is both the maximum of $Y=\left(Y_{1}+Y_{2}\right) / 2$ with respect to joint variation of $\rho_{1}$ and $\rho_{2}$, as well as the local maximum of $Y_{1}$ with respect to $\rho_{1}$ and of $Y_{2}$ with respect to $\rho_{2}$. This universal agreement of the global and local optima is a special feature of the two cell case. If we raise the density of cell one above $1 / 2$, the maximum lossless, propagationless value, then cell one suffers due to its better than $50 \%$ chance of sustaining a hit. Setting the density in cell one to $1 / 2$ decouples the two cells in the optimization problem, so that maximizing the local yield in cell two also optimizes the global yield for the system as a whole.

The specific optimal density assignments do not in general agree for the local and global optima when we consider additional degrees of freedom in the design. However, interestingly we still find that the local and global maxima both correspond to individual cells tuned to one or the other of the specific densities of $1 / 2$ or 1 . In Figs. 2(a1) - 2(a3) we illustrate the global maximum of $Y=\left(Y_{1}+Y_{2}+Y_{3}\right) / 3$, where the probability of a spark in each cell is given by $p_{i}$. We plot our results as a function of $p_{1}$ and $p_{3}$, with $p_{2}=1-p_{1}-p_{3}$. 


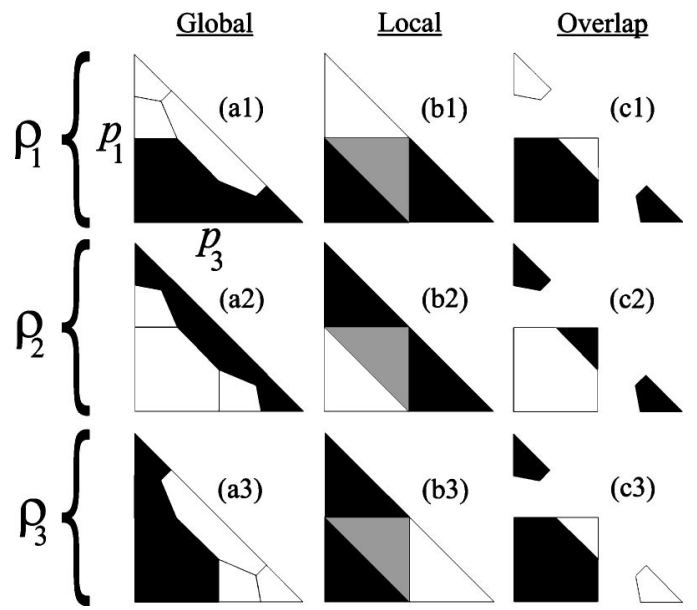

FIG. 2. Optimal solutions for the static case with $N=3$, plotted as a function of $p_{1}$ (vertical axis), and $p_{3}$ (horizontal axis), spanning the full range from 0 to 1 , with $p_{2}=1-p_{1}-p_{3}$. The top row illustrates solutions for cell one, the middle row for cell two, and the bottom row for cell three. Black indicates $\rho_{i}=1$, and white indicates density $\rho_{i}=1 / 2$. Gray indicated degenerate solutions, as described in the text. (a1)-(a3) illustrate the globally optimal solution for each of the three cells. (b1)-(b3) illustrate the locally optimal solutions. (c1)-(c3) indicate the regions where these two solutions overlap.

Separate graphs are used to illustrate the optimal densities $\left[\rho_{1}, \rho_{2}, \rho_{3}\right]$ in each of the cells. White indicates $\rho_{i}=1 / 2$ and black indicates $\rho_{i}=1$.

The three corners of the triangle correspond to cases where one of the $p_{i}$ is equal to 1 , and the other two are zero. Here the optimal solution is clearly $\rho_{i}=1 / 2$ with the other two $\rho_{i}=1$. The rest of the outermost triangular boundary corresponds to regimes where $p_{i}=0$ for one $i$. Even though there are only two cells with a nonzero probability of being hit, these cases are not equivalent to the two cell case, because of the possibility of loss propagating into cell $i$ even if it is never directly hit. The solution is clearly symmetric with respect to interchange of $p_{1}$ and $p_{3}$. Solutions with $p_{2}=1$ are least likely in the phase space, because of the relatively higher potential total losses due to the possibility of nearest neighbor loss through propagation on both sides.

Next we consider local optimization for the three cell case. Because the cells no longer decouple, a configuration which is a maximum of some $Y_{i}$ with respect to the corresponding $\rho_{i}$ will not in general be a maximum for the other two $\left\{Y_{j}, \rho_{j}\right\}$ pairs. Thus to define a local maximum we seek solutions $\left[\rho_{1}, \rho_{2}, \rho_{3}\right]$ which simultaneously maximize the corresponding $\left[Y_{1}, Y_{2}, Y_{3}\right]$. These represent local maxima in two distinct senses. First, we set our criterion for optimal configurations $\left[\rho_{1}, \rho_{2}, \rho_{3}\right]$ based only on a fitness criterion in which $\rho_{i}$ is tuned to maximize $Y_{i}$, rather than the global yield $Y=\left(Y_{1}+Y_{2}+Y_{3}\right) / 3$. Second, for each $Y_{i}$ we seek values of $\rho_{i}$ which locally maximize $Y_{i}\left(\rho_{i}\right)$ in the sense that $\partial Y_{i} / \partial \rho_{i}=0$ and $\partial^{2} Y_{i} / \partial \rho_{i}^{2}<0$, or the appropriate boundary local maximum conditions $\partial Y_{i} / \partial \rho_{i}<0$ at $\rho_{i}=1 / 2$ or $\partial Y_{i} / \partial \rho_{i}>0$ at $\rho_{i}=1$ (which is what we find to be the local maxima in our solutions). Points in phase space which satisfy these criteria would correspond to local attractors for a dynamical system in which the flow is governed by local gradients.

For general functions $\left[Y_{1}, Y_{2}, Y_{3}\right]$, there need be no local optima of the sort we have defined (in which case there would be no stationary fixed points in a dynamical system governed by gradient flows). However, for our case these kinds of triple local optimal solutions (i.e., for $Y_{1}, Y_{2}$, and $Y_{3}$ ) always exist. These are illustrated in Figs. 2(b1)-2(b3). As in the other cases we have considered, all of the optima have each $\rho_{i}$ equal to either $1 / 2$ or 1 . Again black corresponds to $\rho_{i}=1$, white to $\rho_{i}=1 / 2$, and now gray to regions where these are degenerate local optima. For each $i$ there is a local maximum in $Y_{i}\left(\rho_{i}\right)$ at $\rho_{i}=1$ when $p_{i}<1 / 2$, and a local maximum at $\rho_{i}=1 / 2$ when $p_{i}>1 / 2$. There are local maxima at both $\rho_{i}=1 / 2$ and 1 when all three $p_{i}$ are simultaneously less than $1 / 2$. In this case, there are exactly two degenerate solutions (the details are derived in the Appendix) which correspond to $\left[\rho_{1}, \rho_{2}, \rho_{3}\right]=[1,1 / 2,1]$ and $[1 / 2,1,1 / 2]$. Both of these solutions decouple the three cells, and prevent epidemics from spreading.

There are a variety of qualitative and quantitative similarities between the global and local optima. First, the optima are identical over exactly $2 / 3$ of the phase space, as illustrated in Figs. 2(c1)-2(c3). These correspond to regions near the corners of the phase space, where one of the $p_{i}$ significantly outweighs the others, and the center, where there is degeneracy of the local maxima. Thus the qualitative variation of the solutions over the phase space is similar. More importantly, however, the general feature that both local and global optimization select densities of either $1 / 2$ or 1 in each of the cells illustrates a common departure from generic random configurations. Both global and local optimization of the two and three cell cases lead to average yields (for all but the case of exactly equal probabilities) which exceed that associated with the one cell case, which is optimized at criticality. Furthermore, as the number of cells $N=1,2,3$ increases, the yield for the globally optimized case averaged over all possible values of the $p_{i}$ also increases from $1 / 2$ $(N=1)$ to $5 / 8(N=2)$, to $575 / 864(N=3)$ (the average yield for the locally optimized configuration when $N=3$ is $91 /$ 144), indicating increased average fitness with increasing tunable degrees of freedom in the design [19].

While the locally optimized configurations are not always identical to the corresponding global solutions, they do share all the features identified previously in Refs. [9-11] as being common to HOT systems. In all cases, the configurations are highly nongeneric, with high densities and yields, consisting only of densities tuned to $1 / 2$ and 1 in various combinations depending on the probabilities. Both global and local optima are sensitive to changes in the $p_{i}$. This combination of high yields but potential sensitivities to rare events or errors in estimating the relative probabilities of hitting different cells is an example of the "robust, yet fragile" character most essential in HOT. Our systems are too small to exhibit power law distributions, the least general and least important feature of HOT. However, optimization does lead to heavy tails, in this case associated with the fact that in all optimized 
solutions there is at least one cell with the density set to 1 , which insures finite probability of a loss of macroscopic density.

\section{THE DYNAMICS OF REGROWTH}

So far we have focused on static configurations, optimized either globally or locally for yield, with respect to variations in the initial density. Next we consider a generalization of the map, which combines the previous yield map, describing the density in each cell after an infection strikes the system, with a growth map which gives the new density in terms of the old density in any cell which does not sustain a loss. For simplicity, we return to the case of two cells where the global and local spatial optima were equivalent. This also allows us to separate the question of local vs global spatial optimization treated in the previous section from the local vs global maxima in the yield which arise in the time dependent problem. The time dependent map will be generalized to include additional spatial degrees of freedom in future publications.

During a given iteration, the growth map applies only to cells which do not suffer a loss through either a direct hit or the spread of an infection from another cell. The growth map is loosely based on a continuous, deterministic representation of the dynamics of some underlying birth and death process. We will assume this process is confined to each cell individually, so that no cell can be repopulated based on residual densities in other parts of the system.

We model the change in population density with time using an exponential growth map $G\left(\rho_{i}^{(n)}\right)$ which saturates at a maximum density $M_{i}$, which we will take as the design parameter

$$
\rho_{i}^{(n+1)}=G\left(\rho_{i}^{(n)}\right)=\left\{\begin{array}{l}
\alpha \rho_{i}^{(n)} \text { if } \rho_{i}^{(n)} \leqslant M_{i} / \alpha, \\
M_{i} \text { if } \rho_{i}^{(n)} \geqslant M_{i} / \alpha .
\end{array}\right.
$$

Here $\rho_{i}^{(n)}$ represents the density in cell $i$ after $n$ time increments, while $\rho_{i}^{(n+1)}$ represents the density after $n+1$ time increments, assuming the cell is lossless in the time interval between $n$ and $n+1$. In terms of a more complex, spatiotemporal model of population dynamics, $\alpha$ represents the average growth rate of an unsaturated population subject to competing birth and death processes and $M_{i}$ represents the steady state population which results when these processes come into balance. For example, in the contact process [20-22], one of the most well studied spatiotemporal stochastic processes, sites become occupied when neighboring sites give "birth" to offspring, and sites become vacant as a result of "death." We can construct a correspondence in which our parameter $\alpha$ is roughly analogous to the birth rate (since it controls the growth rate at low densities), and, once $\alpha$ is fixed, then $M_{i}$ is determined by the death rate, which leads to the steady state density $\rho_{i}=M_{i}$. On the other hand, when the cell is hit by an infection or when an epidemic propagates into the cell during a particular time increment, the yield maps of the previous section give the density at the end of the interval. The hit map is obtained explicitly by replacing $\rho_{i}$ with $\rho_{i}^{(n)}$ and $Y_{i}$ with $\rho_{i}^{(n+1)}$ in Eqs. (4)-(6).
When we combine the growth model with the loss map, we complete the dynamical system describing the evolution of a population subject to epidemics. Analogous spatiotemporal versions have been considered previously without incorporating design or optimization [23]. Here we consider optimization of the time average yield with respect to the saturation density in the growth map $M_{i}$ for each cell. This is the dynamical analog of the calculation performed in Sec. II.

As before, we find that the yield is optimized both locally and globally when the cell which is hit most often (assumed to be cell one) is at density $1 / 2$. For any growth rate $\alpha$ this corresponds to setting the saturation density $M_{1}$ for that cell equal to $M_{1}=1 / 2$ since this results in no net loss when the cell is infected, thus no net change in the population, so that the balance between birth and death is preserved. Thus, after the saturation density is reached the first time in cell one, it remains fixed at that value for all remaining time (and can thus be ignored). This again decouples the optimization problem for the two cells, so that the local and global spatially optimized solutions are identical.

Setting $\rho_{1}=1 / 2$, the hit map [Eq. (4)] for cell two simplifies, because the only events which result in any net loss in the system are the rare sparks which hit cell two. In this case,

$$
\rho_{2}^{(n+1)}=T\left(\rho_{2}^{(n)}\right)= \begin{cases}\rho_{2}^{(n)}, & \rho_{2}^{(n)} \leqslant 1 / 2, \\ 1-\rho_{2}^{(n)}, & \rho_{2}^{(n)} \geqslant 1 / 2\end{cases}
$$

which describes a simple symmetric tent map $T\left(\rho_{2}^{(n)}\right)$ about $1 / 2$ with slope \pm 1 .

The dynamical system thus simplifies to a composition of Eqs. (7) and (8). Compared to the static case, it is no longer optimal for the second cell to have a density of unity. The growth map presumes that repopulation occurs within the cell, and unit density (achieved through setting the saturation density $M_{2}=1$ ) would result in complete extinction within the cell after the first hit. Furthermore, the larger the density in the second cell, the greater the loss, and thus the longer it takes to repopulate. Optimal solutions for the dynamical problem balance maximizing the density in cell two under the more common circumstances when the first cell is infected, and minimizing the loss due to rare events which infect the second cell.

Initially, we simplify our analysis by removing stochasticity from the sparking process and focusing on a periodic, deterministic, sequence of hits. We assume cell one is hit $N$ times, followed by a single hit on cell two, and then repeat the sequence. This defines the period $N+1$ of the hit map. On average, this corresponds to a relative probability $p$ of hitting cell two, where $p=1 /(N+1)$. For the dynamical map, we optimize the saturation density $M_{2}$ for fixed $N$, assuming fixed growth rate $\alpha>1$. If we allow $\alpha$ to vary, it (trivially) takes the maximum possible value, in order to repopulate the system as rapidly as possible. Thus we fix $\alpha=1.1$, and optimize yield $Y$ (equivalently $Y_{2}$ in the second cell since $Y_{1}$ is fixed at $1 / 2$ ) with respect to the only nontrivial growth parameter parameter $M_{2} \equiv M$.

In Fig. 3 we present a summary of the various dynamical behaviors of the model, plotted in three different ways. Fig- 

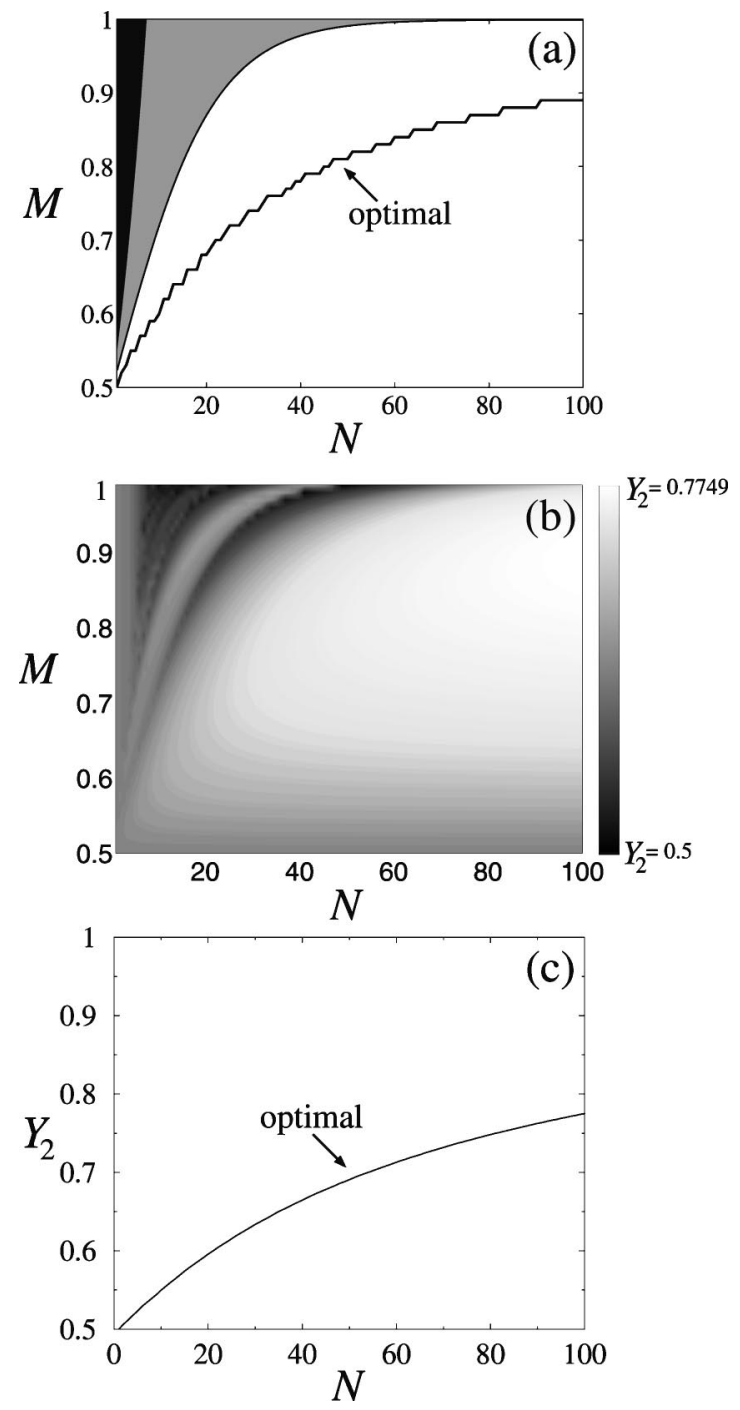

FIG. 3. Dynamical behaviors and optimal solutions for the two cell case with time dependent regrowth. (a) Illustrates the dynamical phase diagram, as a function of the saturation density $M$ of cell two, and the period of the hit map (which is $N+1$ ), with $\alpha=1.1$. The behaviors fall into three distinct regimes: stable fixed points (the white region), periodic solutions (gray), and chaotic solutions (black). The staircase curve which lies within the stable fixed point regime describes optimal values of $M$ for each $N$. (b) illustrates the corresponding values of yield $Y$ in the form of a gray scale contour plot, where black corresponds to the lowest $Y$ values, and white to the highest $Y$ values. (c) illustrates the maximum yield $Y$ as a function of $N$, and corresponds to the $Y$ values on the staircase curve in (a).

ure 3(a) illustrates a phase diagram, plotted as a function of $M$ and $N$, illustrating the different dynamical regimes. The majority of the phase space consists of simple, periodic solutions, in which the period of the density $\rho_{2}^{(n)}$ coincides with the period $N+1$ of the hit map. We will refer to these as stable fixed points, and they occupy the white region in Fig. 3(a). The gray regions corresponds to periodic attractors, in which the period of $\rho_{2}^{(n)}$ is an integer multiple greater than unity of the hit map period $N+1$, and the black region corresponds to chaotic solutions, which are excluded for values of $\alpha$ sufficiently large. The staircase curve which cuts through the stable fixed point portion of the diagram, corresponds to the optimal value of $M$ for each $N$. Figure 3(b) illustrates the values of yield $Y_{2}$ as a function of $M$ and $N$ over the same range shown in Fig. 3(a), where contours of constant shading in the grayscale correspond to constant $Y_{2}$. For each $N$ (a vertical slice of the contour plot), the maximum $Y_{2}$ defines the optimal $M$ values composing the staircase curve in Fig. 3(a). Finally, Fig. 3(c) illustrates the optimal yield $Y_{2}$ as a function of $N$, obtained by optimizing over $M$. These are the $Y_{2}$ values which correspond to the staircase curve describing the optimal $M$ values in Fig. 3(a).

The optimized solutions lie entirely within the stable fixed point region. The fact that the optimal $M$ increases with $N$ in a stepwise manner is a consequence of the discrete nature of our map, and the imposed periodicity of the hit process. To understand the plateaus we assume a stable fixed point solution. Starting from the lowest density $1-M$, define $k$ to be the number of growth steps required to reach the saturation density $M$. The cell will therefore spend $N-k$ steps at the saturation value $M$. This leads to an analytical solution for the yield

$$
\begin{gathered}
Y_{2}=(1-M) \overbrace{\left(1+\alpha+\alpha^{2}+\cdots+\alpha^{k-1}\right.}^{k \text { iterates }})+(N-k) M \\
=(1-M)\left(\frac{\alpha^{k}-1}{\alpha-1}\right)+(N-k) M
\end{gathered}
$$

so that

$$
\begin{aligned}
\frac{\partial Y_{2}}{\partial M}= & -\left(\frac{\alpha^{k}-1}{\alpha-1}\right)+N-k \\
& =N-f(k)
\end{aligned}
$$

where

$$
f(k) \equiv k+\left(\frac{\alpha^{k}-1}{\alpha-1}\right) .
$$

Since $\alpha>1, f(k)$ is a monotonically increasing function of $k$ (equivalently $\partial Y_{2} / \partial M$ is monotonically decreasing with $k$ ).

We are interested in the $M$ which maximizes $Y_{2}$ for a given $N$. Because time is discrete, $k$ takes only integer values, so we are not guaranteed that a solution to $\partial Y_{2} / \partial M$ $=0$ will exist. Instead, the maximum $Y_{2}$ is associated with finding the $M$ which gives rise to a sign change in $\partial Y_{2} / \partial M$, from positive to negative. This corresponds to finding the $k$ for which $f(k-1)<N \leqslant f(k)$. When we find the optimal $k$ which satisfies this inequality, the sign change of $Y_{2}$ at optimality implies $\partial Y_{2} / \partial M \leqslant 0$ for this $k$, so that in order to maximize $Y_{2}$ we choose the minimum $M$ consistent with this value of $k$. But $k$ is the number of iterates before the solution reaches saturation, so by definition $(1-M) \alpha^{k-1} \leqslant M$ which is equivalent to

$$
M \geqslant \frac{1}{1+\alpha^{1-k}} .
$$



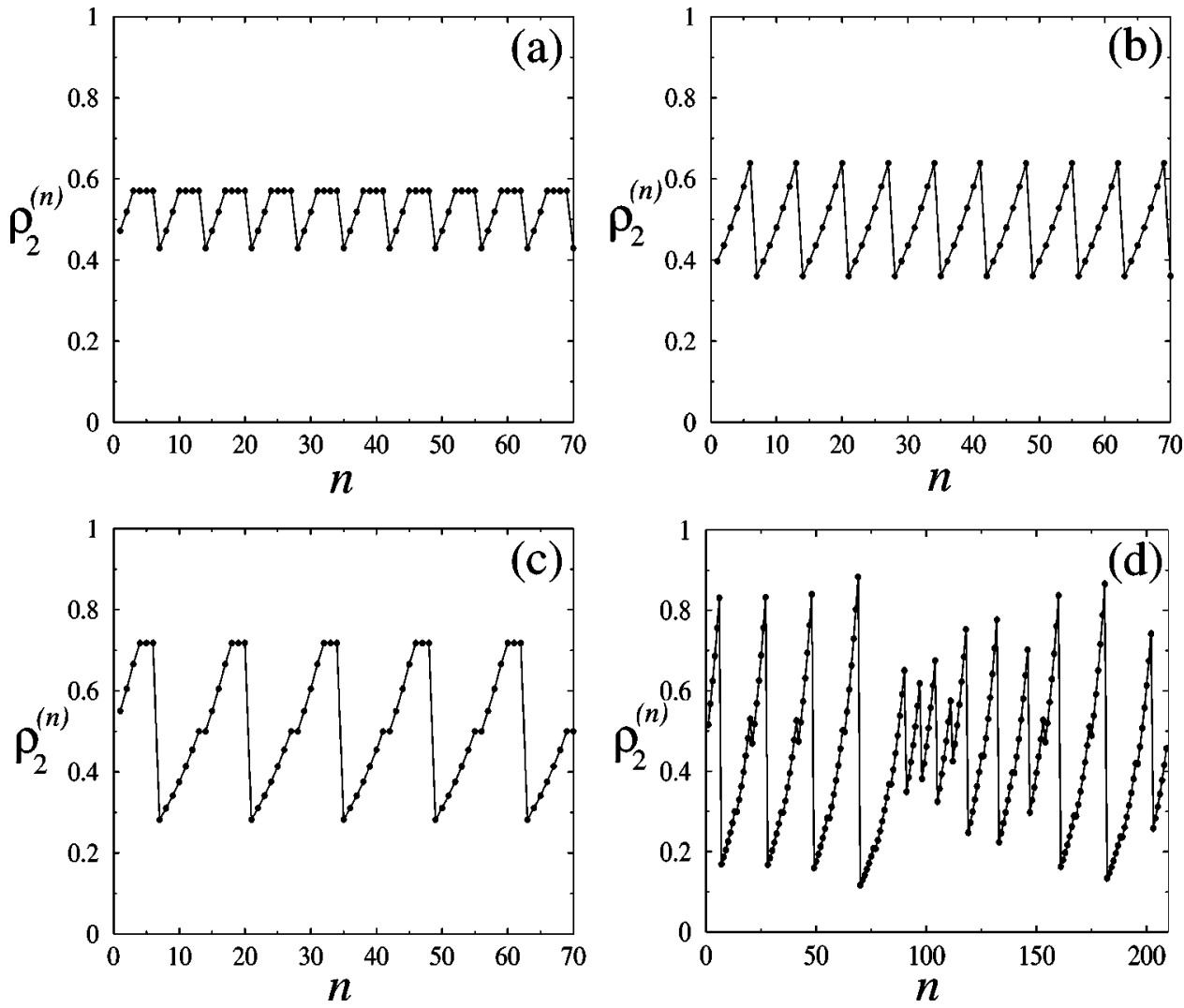

FIG. 4. Solutions for the density $\rho_{2}^{(n)}$ vs time $n$, for $N=6$, and $\alpha=1.1$. In (a) $M=0.571$, which corresponds to the optimal case. In (b) $M=0.6392$, which is the largest value of $M$ for which we still obtain a stable fixed point (i.e., the period of $\rho_{2}^{(n)}$ equals that of the hit map. In (c) $M=0.7177$, which is the highest yield period two solution. In (d) $M=0.9$, which is in the chaotic regime. In this case, the density never hits the saturation value, and the yield is lower than any of the other cases illustrated.
Since the maximum $Y_{2}$ corresponds to the minimum $M$, the optimal $M$ is defined by the case of equality

$$
M=\frac{1}{1+\alpha^{1-k}} .
$$

In the limit of $N \rightarrow \infty, M \rightarrow 1$, and the second cell approaches unit density, which agrees with the results obtained in the static case. Because $M$ is given in terms of the integer values of $k$, and is independent of $N$, we obtain the steps in Fig. 3(a). If we replace the map with a continuous time model, this peculiar behavior does not occur. The corresponding optimal yield does not have a steplike structure, and instead increases smoothly and monotonically with $N$ as shown in Fig. 3(c). In this case, as $N$ increases across a step, additional time is spent at the saturation density, since the number of iterations associated with regrowth remains fixed, increasing the yield, even for fixed $M$.

Other, more complex solutions are possible for the map, though they always correspond to lower average densities than the optimal stable fixed point solution. The gray region in Fig. 3(a) describes a family of periodic solutions of higher periods. For example, a period two solution can be obtained for certain $N$, by choosing $M$ sufficiently high that the growth map requires two periods of the hit map before the density repeats. There is a second, local maximum in the contour plot [Fig. 3(b)], corresponding to the highest yield period two solutions. Chaotic solutions lie within the black wedge in Fig. 3(a). If $\alpha$ is taken sufficiently large, chaotic solutions no longer exist at all. The condition for the existence of chaotic solutions is derived below.
Figures 4 and 5 provide a more detailed picture of these dynamics. Figure 4 illustrates several sample solutions $\rho_{2}^{(n)}$ vs $n$ of the discrete time series. All of the results correspond to one fixed period of the hit map, $N+1=7$, but different values of $M$. We choose this value of $N$, because it illustrates the full range of dynamical behavior, including chaos, along the corresponding vertical slice of the phase diagram [Fig. 3(a)]. Figure 4(a) illustrates a stable fixed point that corresponds to the optimal value of $M$, and thus lies on the staircase of optimal solutions in Fig. 3(a). After three iterations of the growth map, the density saturates, remaining at density $M$ for three more iterates, and then falling to density $1-M$ when cell two is hit, after which the cycle repeats. Figure 4(b) corresponds to a larger value of $M$, which is the largest $M$ for which a period one solution is still observed before the bifurcation to period two. This corresponds to a solution on the boundary between the white and gray regions in Fig. 3(a). Figure 4(c) is the maximum yield period two solution, on the period two crest in the contour plot for yield [Fig. 3(b)]. In Fig. 4(c), beginning with the minimum density of $1-M$, over the first half period the density increases steadily to $1 / 2$. At that point, the cell sustains a hit, but no loss, since the density is at the percolation threshold. Over the second half period, the density continues to grow, reaching saturation after four time increments. Subsequently, the density remains pinned at the saturation value of $M$ before the next hit, which drops the density back to $1-M$, and the cycle repeats. While the maximum density achieved during the cycle (here the value $M$ ) clearly exceeds that of the optimal solution in Fig. 4(a), the average density is less. Finally, Fig. 4(d) corresponds to a value of $M$ which is taken 

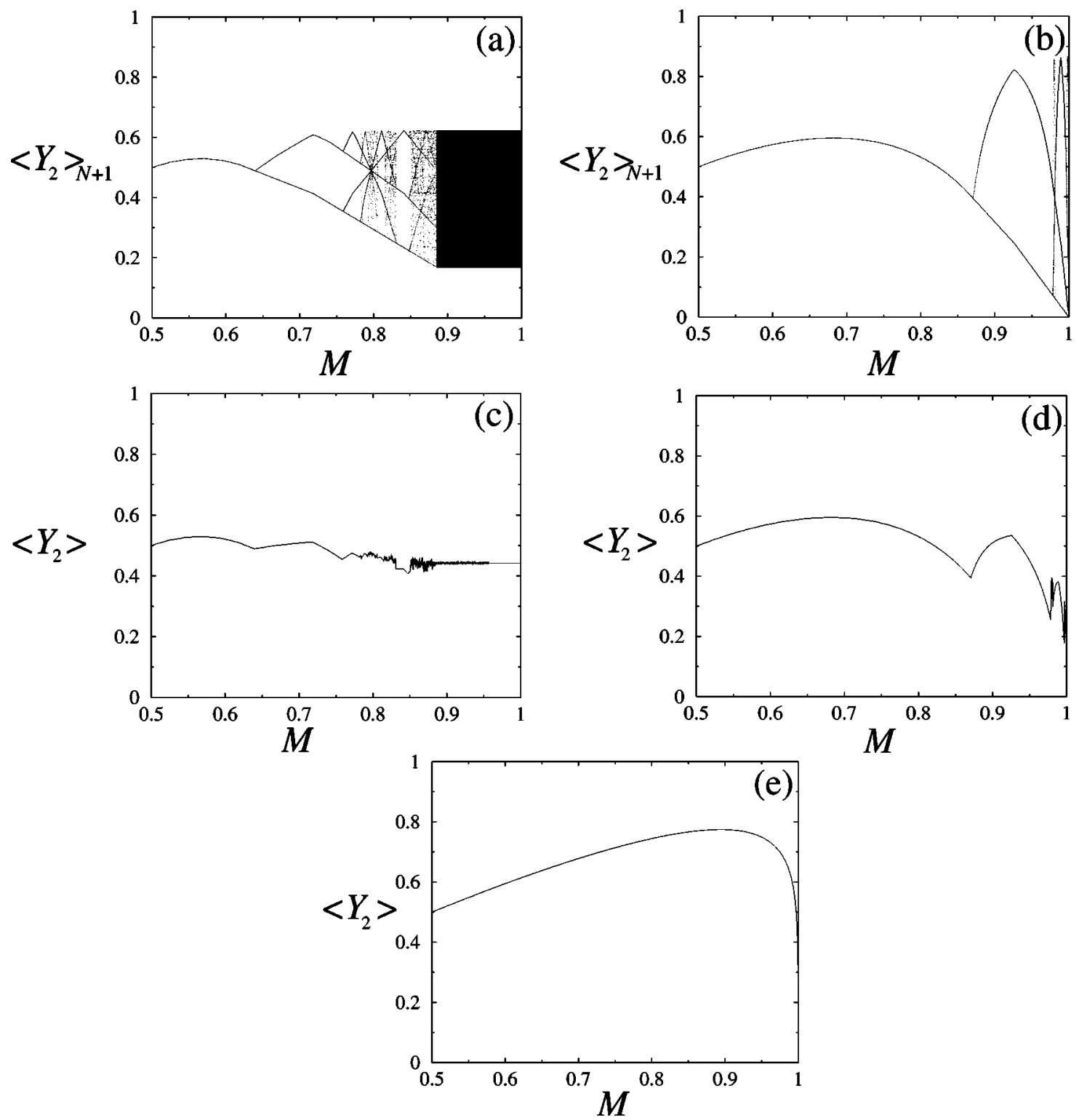

FIG. 5. Bifurcation diagrams and yield curves as a function of $M$ for fixed values of $N$, and $\alpha=1.1$. The bifurcation diagrams illustrate scatter plots of the yield averaged over each single period of the hit map, while the yield curves are the corresponding infinite time averages, from which we can extract the optimal yield and corresponding $M$ value for each case. (a) and (c) are the bifurcation diagram and yield curve, respectively, for $N=6$. (b) and (d) are the bifurcation diagram and yield curve, respectively, for $N=20$. (e) corresponds to $N=100$, where there are stable fixed points for almost all $M$ [up to $M=\left(1.1^{100}\right) /\left(1+1.1^{100}\right)$ ] so there is almost no distinction between the average yield over a single period and the infinite time average.

from the chaotic regime. In this case, the density never repeats and never reaches saturation (if it did it would have to be a periodic solution, rather than chaotic), and the infinite time average yield is extremely low-significantly lower than even the density $\rho=1 / 2$ associated with the percolation threshold.

In Fig. 5 we illustrate a series of bifurcation diagrams [Figs. 5(a), 5(b), and 5(e)] and the corresponding yield plots [Figs. 5(c), 5(d), and 5(e)] as a function of $M$ for fixed $N$. Figs. 5(a) and 5(c), and 5(b) and 5(d) are paired plots for two distinct choices of $N$. Figures 5(a) and 5(c) are obtained for the $N$ value used in Fig. 4. In the case of Fig. 5(e), the bifurcation diagram and the yield plots are essentially indis- tinguishable, so only one plot is included (higher period solutions do exist for this $N$, but they are squeezed so far to the right in the bifurcation diagram that they are not visible). To obtain the bifurcation diagram for a given $N$, we compute the yield $\left\langle Y_{2}\right\rangle_{N+1}$ averaged over each individual period $N+1$ of the hit map. The results are plotted in the form of a scatter plot as a function of $M$, where a stable fixed point corresponds to one point, a period two solution to two points, higher period solutions to more points and eventually a chaotic solution to an infinite number of points in the scatter plot. The corresponding yield plots are simply the infinite time average of the scatter plot data which compose the bifurcation curves, so that the curves are identical for stable 
fixed points [as is essentially the case throughout Fig. 5(e)], the yield is the average of the two branches in the period two regime, and so on. From the maxima of these yield curves we extract the optimal $M$ for each $N$, defining the staircase curve in Fig. 3(a). For the values of $N$ taken for Figs. 5(a)$5(\mathrm{~d})$ the stable fixed point undergoes a series of bifurcations to higher period solutions, and, for $N$ sufficiently small, as in Figs. 5(a)-5(b), there is a bifurcation to chaotic solutions, which are dense, represented by the solid black box in Fig. 5(a).

Comparing Fig. 4, with Figs. 5(a) and 5(c) the optimal solution in Fig. 4(a) corresponds to the maximum yield value in Fig. 5(c), which is on the branch of stable fixed points in Fig. 5(a). The stable fixed point in Fig. 4(b), corresponds to the termination of the stable fixed point branch, at the onset of the bifurcation to period two in Fig. 5(a), and the first local minimum in the yield in Fig. 5(c). Fig. 4(c) correspond to the second local maximum in the yield in Fig. 5(c) (which is slightly kinked-a result of the discrete time dynamics), which is the peak yield period two solution. The period two solutions exist over a range of $M$, given roughly by $[0.63,0.75]$. In Fig. 5(a) we see the two branches of the period two solutions which bifurcate from the period one branch. The values of $\langle Y\rangle_{N+1}$ on the two arms, are the values of $\rho_{2}^{(n)}$ averaged over each period of the hit map required to complete the period two cycle. The upper branch corresponds to the half period which contains the saturation points $\rho_{2}^{(n)}$, and taken alone typically has higher yield than any one of the period one solutions. However, the lower branch, in which the density is rebuilding following the sharp drop to $\rho_{2}^{(n)}=1-M$, always has a sufficiently low average yield that the time average yield of the period two cycle is never as high as the optimized period one solution. Figure 4(d) is embedded in the chaotic regime, which appears solid black in Fig. 5(a), In this case, the density never repeats, and the infinite time average yield is extremely low-significantly lower than the density associated with criticality as illustrated in Fig. 5(c).

For higher values of $N$, the chaotic regime no longer exists. We can analytically determine the phase boundary by standard techniques in dynamical systems theory. The dynamical system which describes $N$ growth cycles in the second cell, followed by a single hit is defined by the composition of the growth and hit map

$$
T \circ G^{N}\left(\rho_{2}^{(n)}\right)= \begin{cases}\alpha^{N} \rho_{2}^{(n)}, & \text { if } \rho_{2}^{(n)} \leqslant \frac{1}{2 \alpha^{N}}, \\ 1-\alpha^{N} \rho_{2}^{(n)}, & \text { if } \frac{1}{2 \alpha^{N}} \leqslant \rho_{2}^{(n)} \leqslant \frac{M}{\alpha^{N}}, \\ 1-M, & \text { if } \rho_{2}^{(n)} \geqslant \frac{M}{\alpha^{N}} .\end{cases}
$$

Figure 6 shows a representation of this map. Note that for $\rho_{2}^{(n)}=1 / 2 \alpha^{N}, T \circ G^{N}\left(1 / 2 \alpha^{N}\right)=1 / 2$, this implies that all iterates of $T \circ G^{N}\left(\rho_{2}^{(n)}\right)$ remain less than $1 / 2$.

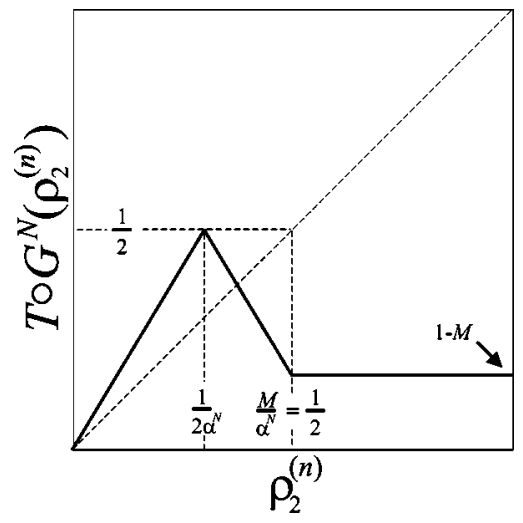

FIG. 6. The composition of $N$ Growth maps $G$ and 1 tent map $T$ vs $\rho_{2}^{(n)}$. If $M / \alpha^{N} \geqslant 1 / 2$ only chaotic solutions are possible throughout the entire interval $\rho_{2} \in(0,1]$. A stable fixed point $\rho_{2}^{*}=1-M$ exists for $M / \alpha^{N} \leqslant 1-M$. This graph is for $0 \leqslant \rho_{2}^{(n)} \leqslant 1$ and 0 $\leqslant T \circ G^{N}\left(\rho_{2}^{(n)}\right) \leqslant 1$.

Since $\alpha>1$, the only stable fixed point is $\rho_{2}^{*}=1-M$. This point only exists for

$$
\frac{M}{\alpha^{N}} \leqslant 1-M,
$$

and its basin of attraction is $\rho_{2} \in(0,1]$. So, at $M=\alpha^{N} /(1$ $\left.+\alpha^{N}\right)+\varepsilon$ (where $\varepsilon$ is a small positive real number), $\rho_{2}^{*}$ loses its stability, and other dynamics are possible.

A key feature of our map is that as soon as one iterate reaches the plateau associated with the saturation density, a stable periodic orbit is inevitable. For an appropriate choice of parameters this can be avoided. Indeed for

$$
\frac{M}{\alpha^{N}} \geqslant 1 / 2,
$$

the dynamics gets trapped in the region $\rho_{2} \leqslant 1 / 2$ and eventually gets trapped in a smaller embedded region

$$
\rho_{2} \in\left[1-\frac{\alpha^{N}}{2}, \frac{1}{2}\right] .
$$

Once confined within this region, the map reduces to a tent map. The Lyapunov exponent $\lambda$ is related to the slope $\alpha^{N}$, leading to $\lambda=N \ln \alpha$. Because $N \geqslant 1$ and $\alpha>1$, we have a positive Lyapunov exponent $\lambda>0$. This implies that for $M / \alpha^{N} \geqslant 1 / 2$, the solutions are chaotic, with no stable periodic orbits allowed.

In Figs. 5(b) and 5(d) we illustrate a bifurcation diagram and the time average yield corresponding to $N=20$ which is a case where high period orbits, but no chaos is observed. As in the case $N=6$, the optimal stable fixed point globally optimizes the yield. The bifurcations to higher period solutions are represented by multiple branches in Fig. 5(b). This leads to additional local optima in yield vs $M$, which have systematically decreasing values of $Y_{2}$ at the local peaks as $M$ increases, also corresponding to increasing period of the solution. 


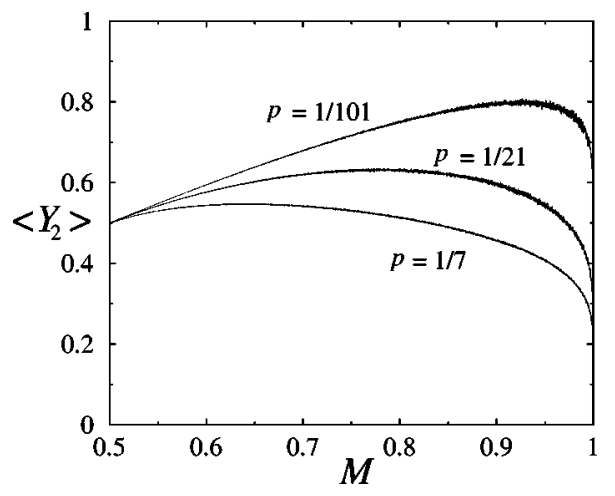

FIG. 7. Yield vs the saturation density $M$ for the stochastic case. We present results which correspond to the same relative probability $p$ for hitting cell two as in the deterministic cases illustrated in Figs. 5(c), 5(d), and 5(e). Compared to the deterministic case, the optimal yields are slightly higher, and all of the structure associated with periodic solutions and chaos is lost.

Figure 5(e) corresponds to a very high period hit map, $N=100$, so that cell two is hit only once every 101 events. In this case, we observe stable fixed point solutions for almost all $M$ [up to $\left.M=\left(1.1^{100}\right) /\left(1+1.1^{100}\right)\right]$, so that the yield $\left\langle Y_{2}\right\rangle_{N+1}$ averaged over one period of the hit map also corresponds almost entirely to the infinite time average. The time average yield in the second cell approaches unity in the limit of large $N$, at a rate which increases with increasing $\alpha$, were $\alpha$ allowed to vary.

Finally, in Fig. 7 we compare our results for the periodic case with the corresponding results for the analogous stochastic map. In the stochastic case, we set $p=1 /(N+1)$, so that with probability $p$ cell two is hit, and with probability $1-p$ regrowth occurs (i.e., cell one is hit, but the density is $1 / 2$ so no loss or propagation occurs as before). This definition of $p$ guarantees that on average the second cell is hit at the same rate as in the corresponding periodic case.

For $p=1 / 7,1 / 21$, and $1 / 101$ (i.e., the stochastic analogs corresponding to the $N$ values in Fig. 5), the stochastic case leads to relatively simpler curves for the time average $Y_{2}$ vs $M$ than we obtained for the deterministic, periodic hit map in Figs. 5(b), 5(d), and 5(e). As illustrated in Fig. 6, in the stochastic case, for each $p$ we observe a single maximum in $Y$ vs $M$, so that all of the structure associated with high period and chaotic solutions is lost. The maximum is shifted to a slightly higher value of $M$ than in the corresponding periodic case. This is easily understood by considering the leading order effect of fluctuations in the hit sequence on the stable fixed point optimal solution [e.g., Fig. 4(a)], which sits at the saturation density $M$, until a hit occurs, at which point it drops to $1-M$, and is subsequently repopulated. If two sequential hits occur relatively closer together than they would in the periodic case, the net loss is less because the density has already dropped, lowering or eliminating the loss compared to the periodic case. On the other hand, if two sequential hits are relatively farther apart, the system is preserved at the saturation density longer. Both of these effects lead to larger average yields for the stochastic case compared to periodic hits.

\section{CONCLUSION}

A variety of low-dimensional dynamical systems have been studied in the context of population dynamics [24]. Examples include the classic Lotka-Volterra equations which describe competing populations in ecology [25], as well as the logistic map [26] which attempts to describe complexities which can arise within an individual population. Similar to ours, these maps can exhibit rich and varied dynamical behavior, including periodic and chaotic solutions. However, these and other previous studies have focused primarily on the dynamics which arise as a consequence of internal interactions within a community, ignoring interactions of the community with the external environment. Our model combines the study of a relatively simpler dynamical system describing the isolated community (the exponential growth and saturation map), with a coupling to external perturbations. The resulting map can exhibit complex solutions, though the high yield HOT states are always confined to the simplest regime in which the period of the solution is the same as the period associated with external perturbations.

We should mention the following result obtained in Refs. [27-29]: "Optimal periodic orbits of chaotic systems occur at low period.' This result is restricted to the chaotic regime. We are interested in the whole picture, stable orbits included. In our case, the optimal periodic orbit for the whole dynamical regime is also a low period orbit (a fixed point), but a stable orbit away from the chaotic regime.

In constructing the map we had in mind a coarse-grained representation of an underlying many-degree-of-freedom percolation model or contact process, in which a birth and death process governs repopulation of individual cells, and infections spread rapidly throughout connected clusters. Indeed, using a microscopic description of this kind we have begun to perform preliminary studies of the issues discussed in this paper. In that case, optimal design corresponds to tuning the local birth and death rates based on global or local yield criteria. With design, it is possible to obtain solutions which significantly outperform uniform or random systems, although compared to our simple map, these simulations take much longer to converge.

It would also be useful to explore in detail the sensitivities of our results to the features of the map we have defined. In this context, it would be of particular interest to incorporate optimization and coupling to external perturbations in models traditionally used in ecology. In ecological modeling, stabilizing features are occasionally put in by hand to more fully capture realistic biological phenomena and structured interactions. Could such features arise from robustness? Furthermore, environmental impact studies focus on sensitivity of systems to environmental change, corresponding loosely to a nonstationary $P(i, j)$. If ecosystems are HOT, and therefore tuned for robustness in a manner which reflects their historical environmental conditions, then they may be hypersensitive to change in a manner which is not accurately reflected in models which do not take the synergy between the system and its environment into account. 


\section{ACKNOWLEDGMENTS}

We wish to thank T. Zhou for numerous useful discussions. This work was supported by the David and Lucile Packard Foundation, and NSF Grant No. DMR-9813752, and EPRI/DoD through the Program on Interactive Complex Networks.

\section{APPENDIX}

In this Appendix, we present a more detailed description of the one, two, and three cell maps discussed in Sec. II. We also give the explicit forms of the global and local yield functions for the three cell case. We derive the local optimal solutions for the three cell degenerate case (Fig. 2), as well as the local and global optimal solution for the two cell case, which is always $\left[\rho_{1}, \rho_{2}\right]=[1 / 2,1]$.

\section{Detailed one, two, and three cell maps} by

The one-dimensional (1D) hit map (static case) is given

$$
Y_{1}=\rho_{1}-P_{\infty}^{2}\left(\rho_{1}\right)
$$

Similarly, the 2D hit map (static case) is given by

$$
\begin{aligned}
& \text { if hit cell one: }\left\{\begin{array}{l}
Y_{1}=\rho_{1}-P_{\infty}^{2}\left(\rho_{1}\right), \\
Y_{2}=\rho_{2}-P_{\infty}\left(\rho_{1}\right) P_{\infty}^{2}\left(\rho_{2}\right),
\end{array}\right. \\
& \text { if hit cell two: }\left\{\begin{array}{l}
Y_{1}=\rho_{1}-P_{\infty}\left(\rho_{2}\right) P_{\infty}^{2}\left(\rho_{1}\right), \\
Y_{2}=\rho_{2}-P_{\infty}^{2}\left(\rho_{2}\right),
\end{array}\right.
\end{aligned}
$$

Notice that these equations are symmetric. Indeed, we can replace $\rho_{1}$ by $\rho_{2}$, and $\rho_{2}$ by $\rho_{1}$ in Eq. (A2) and we get Eq. (A3).

A similar treatment for the three cells case yields

$$
\begin{aligned}
& \text { if hit cell one: }\left\{\begin{array}{c}
Y_{1}=\rho_{1}-P_{\infty}^{2}\left(\rho_{1}\right), \\
Y_{2}=\rho_{2}-P_{\infty}\left(\rho_{1}\right) P_{\infty}^{2}\left(\rho_{2}\right), \\
Y_{3}=\rho_{3}-P_{\infty}\left(\rho_{1}\right) P_{\infty}\left(\rho_{2}\right) P_{\infty}^{2}\left(\rho_{3}\right),
\end{array}\right. \\
& \text { if hit cell two: }\left\{\begin{array}{c}
Y_{1}=\rho_{1}-P_{\infty}\left(\rho_{2}\right) P_{\infty}^{2}\left(\rho_{1}\right), \\
Y_{2}=\rho_{2}-P_{\infty}^{2}\left(\rho_{2}\right), \\
Y_{3}=\rho_{3}-P_{\infty}\left(\rho_{2}\right) P_{\infty}^{2}\left(\rho_{3}\right),
\end{array}\right. \\
& \text { if hit cell three: }\left\{\begin{array}{c}
Y_{1}=\rho_{1}-P_{\infty}\left(\rho_{3}\right) P_{\infty}\left(\rho_{2}\right) P_{\infty}^{2}\left(\rho_{1}\right), \\
Y_{2}=\rho_{2}-P_{\infty}\left(\rho_{3}\right) P_{\infty}^{2}\left(\rho_{2}\right), \\
Y_{3}=\rho_{3}-P_{\infty}^{2}\left(\rho_{3}\right) .
\end{array}\right.
\end{aligned}
$$

\section{Global and local yields}

The total yield in the three cell case is given by

$$
\begin{aligned}
Y= & p_{1}\left[\rho_{1}+\rho_{2}+\rho_{3}-P_{\infty}^{2}\left(\rho_{1}\right)-P_{\infty}\left(\rho_{1}\right) P_{\infty}^{2}\left(\rho_{2}\right)\right. \\
& \left.-P_{\infty}\left(\rho_{1}\right) P_{\infty}\left(\rho_{2}\right) P_{\infty}^{2}\left(\rho_{3}\right)\right]+p_{2}\left[\rho_{1}+\rho_{2}+\rho_{3}\right. \\
& \left.-P_{\infty}\left(\rho_{2}\right) P_{\infty}^{2}\left(\rho_{1}\right)-P_{\infty}^{2}\left(\rho_{2}\right)-P_{\infty}\left(\rho_{2}\right) P_{\infty}^{2}\left(\rho_{3}\right)\right] \\
& +p_{3}\left[\rho_{1}+\rho_{2}+\rho_{3}-P_{\infty}\left(\rho_{3}\right) P_{\infty}\left(\rho_{2}\right) P_{\infty}^{2}\left(\rho_{1}\right)\right. \\
& \left.-P_{\infty}\left(\rho_{3}\right) P_{\infty}^{2}\left(\rho_{2}\right)-P_{\infty}^{2}\left(\rho_{3}\right)\right] .
\end{aligned}
$$

The yield in cell one, cell two, and cell three, respectively, are

$$
\begin{aligned}
Y_{1}= & p_{1}\left[\rho_{1}-P_{\infty}^{2}\left(\rho_{1}\right)\right]+p_{2}\left[\rho_{1}-P_{\infty}\left(\rho_{2}\right) P_{\infty}^{2}\left(\rho_{1}\right)\right] \\
& +p_{3}\left[\rho_{1}-P_{\infty}\left(\rho_{3}\right) P_{\infty}\left(\rho_{2}\right) P_{\infty}^{2}\left(\rho_{1}\right)\right], \\
Y_{2}= & p_{1}\left[\rho_{2}-P_{\infty}\left(\rho_{1}\right) P_{\infty}^{2}\left(\rho_{2}\right)\right]+p_{2}\left[\rho_{2}-P_{\infty}^{2}\left(\rho_{2}\right)\right] \\
& +p_{3}\left[\rho_{2}-P_{\infty}\left(\rho_{3}\right) P_{\infty}^{2}\left(\rho_{2}\right)\right], \\
Y_{3}= & p_{1}\left[\rho_{3}-P_{\infty}\left(\rho_{1}\right) P_{\infty}\left(\rho_{2}\right) P_{\infty}^{2}\left(\rho_{3}\right)\right] \\
& +p_{2}\left[\rho_{3}-P_{\infty}\left(\rho_{2}\right) P_{\infty}^{2}\left(\rho_{3}\right)\right]+p_{3}\left[\rho_{3}-P_{\infty}^{2}\left(\rho_{3}\right)\right] .
\end{aligned}
$$

\section{Derivation of the optima in the gray region of Fig. 2}

The gray region of Fig. 2 is characterized by

$$
p_{1}<1 / 2, \quad p_{2}<1 / 2, \quad \text { and } p_{3}<1 / 2 \text {. }
$$

We also impose

$$
p_{1}+p_{2}+p_{3}=1
$$

It is easy to prove that the triplets $(1 / 2,1,1 / 2)$ and $(1,1 / 2,1)$ are degenerate local optima in this region. For example, for $(1 / 2,1,1 / 2)$, we substitute this solution into $\partial Y_{i} / \partial \rho_{i}$ and using Eqs. (A11) and (A12), we obtain

$$
\begin{gathered}
\partial Y_{1} / \partial \rho_{1}=-p 1-p 2+p 3<0, \\
\partial Y_{2} / \partial \rho_{2}=p 1-p 2+p 3>0, \\
\partial Y_{3} / \partial \rho_{3}=p 1-p 2-p 3<0 .
\end{gathered}
$$

This proves that $(1 / 2,1,1 / 2)$ is a local optimum. A similar procedure illustrates the corresponding results for $(1,1 / 2,1)$. No other triplet satisfies the conditions for being an optimum. For example, trivially, the triplet $(1,1,1)$ can never be an optimal solution for any $\left[p_{1}, p_{2}, p_{3}\right]$, since it always produced zero yield. Also, we can easily verify the triplet $(1 / 2,1 / 2,1 / 2)$ is never optimal:

$$
\begin{gathered}
\partial Y_{1} / \partial \rho_{1}=1-2 p_{1}, \\
\partial Y_{2} / \partial \rho_{2}=1-2 p_{2}, \\
\partial Y_{3} / \partial \rho_{3}=1-2 p_{2} .
\end{gathered}
$$


Optimality requires that (A16), (A17), and (A18) all be less than 0 . This would require all $p_{i}>1 / 2$, which is impossible. Therefore, $(1 / 2,1 / 2,1 / 2)$ is never an optimum.

\section{Derivation of optimal solution $\left[\rho_{1}, \rho_{2}\right]=[1 / 2,1]$ in the static two cell case}

If $\rho_{1} \geqslant 1 / 2$ and $\rho_{2} \geqslant 1 / 2$, the yield is

$$
\begin{aligned}
Y= & (1-p)\left[1-\rho_{2}+\rho_{1}-\sqrt{2 \rho_{2}-1}\left(2 \rho_{1}-1\right)\right] \\
& +p\left[1-\rho_{2}+\rho_{1}-\sqrt{2 \rho_{2}-1}\left(2 \rho_{1}-1\right)\right],
\end{aligned}
$$

and this surface in 3D space has a maximum for $\rho_{1}=1 / 2$ and $\rho_{2}=1$ giving $Y=3 / 2-p$. If $\rho_{1} \leqslant 1 / 2$ and $\rho_{2} \geqslant 1 / 2$, then

$$
\begin{aligned}
Y & \left.=(1-p)\left[\rho_{1}+\rho_{2}\right]+p\left[1-\rho_{2}+\rho_{1}\right]\right) \\
& =\rho_{1}+\left(1-2 p \rho_{2}\right)+p
\end{aligned}
$$

This takes a maximum value of $3 / 2-p$ for $\rho_{1}=1 / 2$ and $\rho_{2}$ $=1$. Finally, if $\rho_{1} \geqslant 1 / 2$ and $\rho_{2} \leqslant 1 / 2$, then

$$
Y=(1-p)\left[1-\rho_{1}+\rho_{2}\right]=1-p+(2 p-1) \rho_{1}+\rho_{2}=1
$$

for $\rho_{1}=1 / 2$ and $\rho_{2}=1 / 2$. Therefore, we get the two optimal densities of $\rho_{1}=1 / 2$ and $\rho_{2}=1$ giving an optimal yield of $Y=3 / 2-p$.
[1] P. Bak, C. Tang, and K. Wiesenfeld, Phys. Rev. Lett. 59, 381 (1987).

[2] P. Bak, How Nature Works: The Science of Self-Organized Criticality (Copernicus, New York, 1996).

[3] S.A. Kauffman, The Origins of Order: Self-Organization and Selection in Evolution (Oxford University Press, New York, 1993).

[4] S. Levin, Fragile Dominion (Perseus Books, Boston, 1999).

[5] K. McCann, A. Hastings, and G. Huxel, Nature (London) 395, 794 (1998).

[6] I. Hanski, Nature (London) 390, 440 (1997).

[7] S.L. Pimm, Ecology 61, 219 (1980).

[8] P. Morin and S. Lawler, Annu. Rev. Ecol. Syst. 26, 505 (1995).

[9] J.M. Carlson and J. Doyle, Phys. Rev. Lett. 84, 2529 (2000); Phys. Rev. E 60, 1412 (1999).

[10] J. Doyle and J.M. Carlson, Phys. Rev. Lett. 84, 5656 (2000).

[11] T. Zhou and J.M. Carlson, Phys. Rev. E 62, 3197 (2000).

[12] R.E. Ulanowicz, Ecology, the Ascendent Perspective (Columbia University Press, New York, 1997).

[13] S.A. Levin, Ecology 73, 1943 (1992).

[14] W. Swenson, D.S. Wilson, and R. Elias, Proc. Natl. Acad. Sci. U.S.A. 97, (2000).

[15] E. Sober and D.S. Wilson, The Evolution of Altruism (Harvard University Press, Cambridge, 1998).

[16] D. Stauffer and A. Aharony, Introduction to Percolation Theory, 2nd ed. (Taylor \& Francis, London, 1992).

[17] K. Chen, P. Bak, and M.H. Jensen, Phys. Lett. A 149, 207
(1990); B. Drossel, and F. Schwabl, Phys. Rev. Lett. 69, 1629 (1992).

[18] J.H. Brown, Macroecology (University of Chicago Press, Chicago, 1995).

[19] Similar results are obtained directly for a system with many spatial degrees of freedom. J.M. Carlson and J. Doyle (unpublished).

[20] The contact process is a stochastic process of a population dynamics with birth rates depending on the number and proximity of neighbors and death rates. T.E. Harris, Ann. Prob. 2, 969 (1974).

[21] For a review of the contact process see R. Durrett and S.A. Levin, Philos. Trans. R. Soc. London, Ser. B 343, 329 (1994).

[22] For an introduction to Stochastic Processes see R. Durrett, Essentials of Stochastic Processes (Springer, New York, 1999).

[23] S.A. Richards, W.G. Wilson, and J.E.S. Socolar, Philos. Trans. R. Soc. London, Ser. B 266, 2383 (1999).

[24] W. Gurney and R. Nisbet, Ecological Dynamics (Oxford University Press, New York, 1998).

[25] A.J. Lotka, Elements of Mathematical Biology (Dover Publications, New York, 1956). (This is a reprint of the 1924 classic, Elements of Mathematical Biology, by A.J. Lotka, originally published by the Williams and Wilkins Co.)

[26] R.M. May, Nature (London) 261, 459 (1976).

[27] B.R. Hunt and E. Ott, Phys. Rev. Lett. 76, 2254 (1996); Phys. Rev. E 54, 328 (1996).

[28] G. Yuan and B.R. Hunt, Nonlinearity 12, 1207 (1999).

[29] T.H. Yang, B.R. Hunt, and E. Ott, Phys. Rev. E 62, 1950 (2000). 